\title{
Adaptive Sliding Mode Control with Backstepping Approach for a Moving Mass Hypersonic Spinning Missile
}

\author{
Feng-Jie $\mathrm{GAO}^{1,}$, , Liang-Yu ZHAO ${ }^{1,2, \mathrm{~b}}$ \\ ${ }^{1}$ School of Aerospace Engineering, Beijing Institute of Technology, Beijing 100081, China \\ ${ }^{2}$ Key Laboratory of Dynamics and Control of Flight Vehicle, Ministry of Education, \\ Beijing 100081, China \\ afengjie_gao@163.com, b zhaoly@bit.edu.cn (Corresponding Author)
}

Keywords: adaptive sliding mode control; backstepping; spinning missile; moving mass.

\begin{abstract}
The dynamics model of a hypersonic spinning missile with two moving masses is established. Considering the inherent uncertainties of the mathematical model and the aerodynamic parameters, an affine model with external disturbances is established and an attitude adaptive sliding mode controller is designed following the backstepping approach. The stability of the closed-loop system is proved by means of Lyapunov method. A saturation function is introduced to eliminate the chattering of the sliding mode controller, and the numerical simulations show that the attitude controller is effective.
\end{abstract}

\section{Introduction}

Moving mass control generates the control torque by changing the positions of the internal masses to control the attitude of the aircraft [1]. Compared with the pneumatic rudder control, moving mass control can ensure the aerodynamic configuration of the aircraft, and reduce the aerodynamic drag and the rudder surface ablation [2], which is why the moving mass control is very suitable to hypersonic vehicles. A kinetic energy interceptor was well controlled via moving mass control [1]. The influence of moving masses on the rolling speed of a spinning missile was studied in [3]. When the moving mass control technique is employed to control a hypersonic spinning missile, both the radial control and the axial control of the centroid offset should be conducted [3]. As shown in Fig.1, there are two moving masses ( $p$ mass and $q$ mass) which are located along the axial direction and the radial direction, respectively.

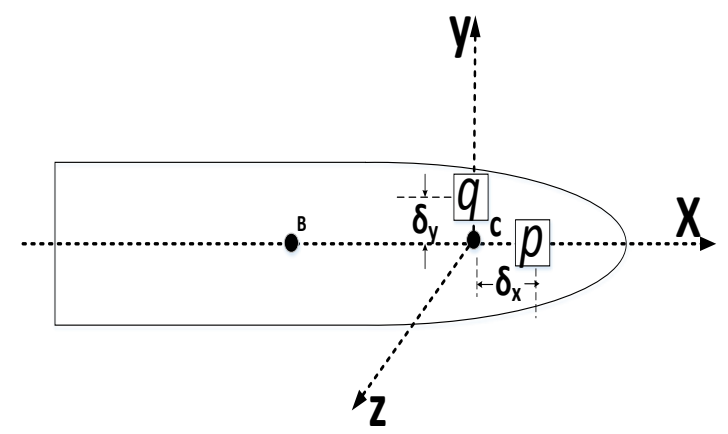

Fig. 1 Illustriton of the spinning missile and the moving masses

Due to the strong couplings, nonlinearities and model uncertainties of moving mass spinning missiles, the design of an attitude controller needs advanced approaches [4, 5]. In this paper, based on backstepping approach, the system is divided into two subsystems. An adaptive sliding mode control is used to design subsystem 1 , and the sliding mode surface parameters can change adaptively. A global fixed time convergent sliding mode surface is used to design subsystem 2, which makes subsystem 2 always stay near the sliding surface from the initial time. Simulation results show that the attitude control system can track the commands within a given fixed time. 


\section{Dynamics Model}

Following the Newtonian mechanics and small disturbance assumption, the dynamics model of a spinning missile shown in Fig. 1 can be established as,

$$
\begin{aligned}
& {\left[\begin{array}{c}
\dot{\alpha} \\
\dot{\beta}
\end{array}\right]=\left[\begin{array}{cc}
\sin \alpha \tan \beta & 1 \\
\cos \alpha & 0
\end{array}\right]\left[\begin{array}{c}
\omega_{y 4} \\
\omega_{z 4}
\end{array}\right]+\left[\begin{array}{c}
\frac{-R_{x} \sin \alpha+R_{y} \cos \alpha}{V m_{s} \cos \beta} \\
\frac{\left(-R_{x} \cos \alpha+R_{y} \sin \alpha\right) \sin \beta+R_{z} \cos \beta}{V m_{s}}
\end{array}\right] .} \\
& {\left[\begin{array}{c}
\dot{\omega}_{y 4} \\
\dot{\omega}_{z 4}
\end{array}\right]=\left[\begin{array}{cc}
\frac{m_{y}^{\omega_{y}} q s L^{2}}{V J_{2}} & \frac{-J_{1} \dot{\gamma}}{J_{2}} \\
\frac{J_{1} \dot{\gamma}}{J_{2}} & \frac{m_{z}^{\omega_{z}} q s L^{2}}{V J_{2}}
\end{array}\right]\left[\begin{array}{l}
\omega_{y 4} \\
\omega_{z 4}
\end{array}\right]+\left[\begin{array}{cc}
\frac{u_{1} R_{z}}{J_{2}} & \frac{-u_{2} R_{x} \sin \gamma}{J_{2}} \\
\frac{-u_{1} R_{y}}{J_{2}} & \frac{u_{2} R_{x} \cos \gamma}{J_{2}}
\end{array}\right]\left[\begin{array}{l}
\delta_{x} \\
\delta_{y}
\end{array}\right]+\left[\begin{array}{cc}
\frac{-m_{u} q s L^{2} \dot{\gamma}}{V J_{2}} & \frac{m_{y}^{\beta} q s L-q s C_{z}^{\beta}\left(u_{1}+u_{2}\right) l}{J_{2}} \\
\frac{m_{z}^{\alpha} q s L}{J_{2}} & \frac{m_{u} q s L^{2} \dot{\gamma}+q s C_{z}^{\beta}\left(u_{1}+u_{2}\right) l}{V J_{2}}
\end{array}\right]\left[\begin{array}{l}
\alpha \\
\beta
\end{array}\right] .}
\end{aligned}
$$

where $q=0.5 \rho V^{2}$ is the dynamic press, $\rho$ is the air density, $S$ is the reference area. $m_{p}, m_{q}$ are the mass of $p$ and $q$, respectively, the total mass of spinning missile is $m_{s}, u_{1}=m_{p} / m_{s}, u_{2}=m_{q} / m_{s}$ are mass ratios, $\omega_{4}=\left[\begin{array}{lll}\omega_{x 4} & \omega_{y 4} & \omega_{z 4}\end{array}\right]$ is the rotational angular velocity of the quasi body coordinate system relative to the ground coordinate system, $\boldsymbol{R}_{4}=\left[\begin{array}{lll}R_{x} & R_{y} & R_{z}\end{array}\right]^{T}$ is the air force in the quasi body coordinate system, $\delta_{x}, \delta_{y}$ are the shifts of $p$ and $q$, respectively; $m_{z}^{\alpha}$ and $m_{y}^{\beta}$ are the static torque coefficients which are relative to the angle of attack and sideslip, respectively. $m_{x}^{\omega_{x}}, m_{y}^{\omega_{y}}, m_{z}^{\omega_{z}}$ are damping moment coefficients, $m_{u}$ is the Magnus coefficient, $\dot{\gamma}$ is the differential of roll angle, $L$ is the characteristic length, $l$ is the distance from the centroid to $C$ as shown in Fig.1.

Define the state variable $\boldsymbol{x}_{1}=\left[\begin{array}{ll}\alpha & \beta\end{array}\right]^{T}, \boldsymbol{x}_{2}=\left[\begin{array}{ll}\omega_{y 4} & \omega_{z 4}\end{array}\right]^{T}$, the control variable $\boldsymbol{u}=\left[\begin{array}{ll}\delta_{x} & \delta_{y}\end{array}\right]^{T}$. Taking the uncertainties of the control system into consideration, Eq.(1) and Eq.(2) can be rewritten as:

$$
\begin{aligned}
& \dot{x}_{1}=\boldsymbol{f}_{1}\left(\boldsymbol{x}_{1}\right)+\boldsymbol{g}_{1}\left(\boldsymbol{x}_{1}\right) \boldsymbol{x}_{2}+\Delta \boldsymbol{f}_{1}+\boldsymbol{d}_{\mathbf{1}} . \\
& \dot{\boldsymbol{x}}_{2}=\boldsymbol{f}_{2}\left(\boldsymbol{x}_{1}, \boldsymbol{x}_{2}\right)+\boldsymbol{g}_{2}\left(\boldsymbol{x}_{1}, \boldsymbol{x}_{2}\right) \boldsymbol{u}+\Delta \boldsymbol{f}_{2}+\boldsymbol{d}_{2} .
\end{aligned}
$$

where $\left\|\boldsymbol{d}_{i}\right\|<h_{i},\left\|\Delta \boldsymbol{f}_{i}\right\|<p_{i},(i=1,2), h_{i}$ and $p_{i}$ are positive.

\section{Design of the Controller}

Following the backstepping approach, the attitude control system can be divided into two subsystems as shown in Fig.2. Subsystem 1 is the attitude angles tracking loop, and $\boldsymbol{x}_{2}$ is the virtual control variable, $\boldsymbol{x}_{1}$ the output. Subsystem 2 is the attitude angular velocities tracking loop, and the input is $\boldsymbol{u}$, the output is the virtual control variable $\boldsymbol{x}_{2}$ of the subsystem 1 .

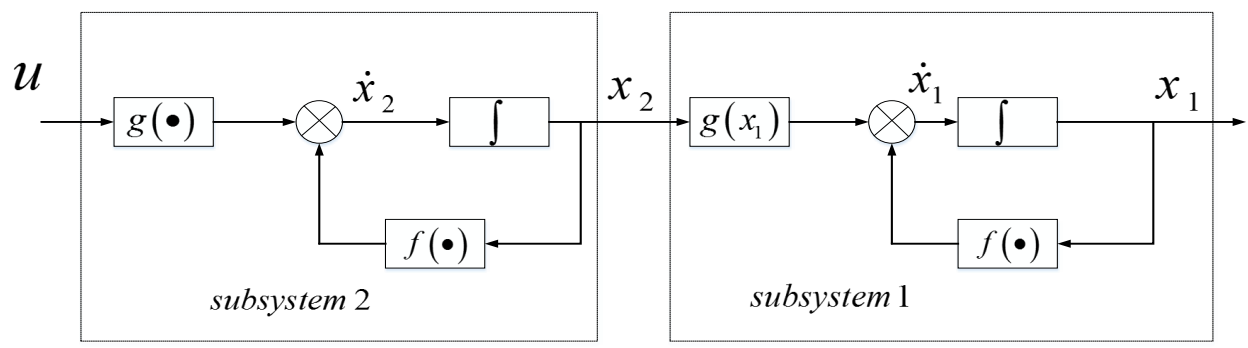

Fig. 2 The control system and its two subsystems 
Firstly, for the subsystem 1 , define the tracking error $\boldsymbol{z}_{1}=\boldsymbol{x}_{1}-\boldsymbol{x}_{1 d}$. An adaptive sliding mode surface with integral is selected as:

$$
\boldsymbol{S}_{1}=\mathbf{z}_{1}+k(t) \int_{0}^{t} \mathbf{z}_{1}(\tau) d \tau
$$

where $k(t)$ changes with time adaptively, and its initial value is $k_{1} \cdot \boldsymbol{x}_{2 d}$ is set as the control command of subsystem 1 . Then the virtual control variable of subsystem 1 is

$$
\boldsymbol{x}_{2 d}=-\boldsymbol{g}_{1}\left(\boldsymbol{x}_{1}\right)^{-1}\left[\boldsymbol{f}_{1}\left(\boldsymbol{x}_{1}\right)+k(t) \mathbf{z}_{1}+\eta_{1} \mathbf{s}_{1}-\dot{\boldsymbol{x}}_{1 d}\right]
$$

where $\eta_{1}$ is the switching gain, and it should satisfy $\eta_{1}>\left(p_{1}+h_{1}\right)$, the adaptive rate is $\dot{k}(t)=-\mathbf{s}_{1} \int_{0}^{t} \mathbf{z}_{1} d \tau$.

Consider the Lyapunov function $V_{1}=\frac{1}{2} \boldsymbol{s}_{1}^{T} \boldsymbol{s}_{1}$, its derivative is

$$
\dot{\boldsymbol{V}}_{1}=\boldsymbol{s}_{1}{ }^{T}\left(-\eta_{1} \boldsymbol{s}_{1}+\Delta \boldsymbol{f}_{1}+\boldsymbol{d}_{1}-\boldsymbol{s}_{1}\left(\int_{0}^{t} \mathbf{z}_{1} d \tau\right)^{2}\right) \leq\left|\boldsymbol{s}_{1}\right|\left[-\eta_{1}+\left(h_{1}+p_{1}\right)\right]-\boldsymbol{s}_{1}^{T} \boldsymbol{s}_{1}\left(\int_{0}^{t} \mathbf{z}_{1} d \tau\right)^{2} \leq 0 .
$$

This means the subsystem 1 is stable.

Secondly, for the subsystem 2, the tracking signal $\boldsymbol{x}_{2 d}$ is the control command of subsystem 1 . The error vector is $\boldsymbol{z}_{2}=\boldsymbol{x}_{2}-\boldsymbol{x}_{2 d}$, the global fixed time convergent sliding mode surface is,

$$
\boldsymbol{s}_{2}=\mathbf{z}_{2}+\boldsymbol{w}(t)+k_{2} \int_{0}^{t}\left(\mathbf{z}_{2}+\boldsymbol{w}(t)\right) d \tau
$$

where $k_{2}$ is constant, and the convergent time $t$ can be freely given by the designer. The control variable of subsystem $2 \boldsymbol{u}$ is calculated by,

$$
\boldsymbol{u}=-\boldsymbol{g}_{2}^{-1}\left(\boldsymbol{x}_{1}, \boldsymbol{x}_{2}\right)\left(\boldsymbol{f}_{2}\left(\boldsymbol{x}_{1}, \boldsymbol{x}_{2}\right)+\eta_{2} \operatorname{sgn}\left(\boldsymbol{s}_{2}\right)-\dot{\boldsymbol{x}}_{2 d}+\dot{\boldsymbol{w}}(t)+k_{2} \mathbf{z}_{2}+k_{2} \boldsymbol{w}(t)\right) .
$$

where $\eta_{2}$ is the switching gain, and it should satisfy $\eta_{2}>\left(p_{2}+h_{2}\right)$.

Consider the Lyapunov function $V_{2}=\frac{1}{2} \boldsymbol{s}_{2}{ }^{T} \boldsymbol{s}_{2}$, its derivative is

$$
\dot{\boldsymbol{V}}_{2}=\boldsymbol{s}_{2}^{T}\left(-\eta_{2} \operatorname{sgn}\left(\boldsymbol{s}_{2}\right)+\Delta \boldsymbol{f}_{2}+\boldsymbol{d}_{2}\right) \leq\left|\boldsymbol{s}_{2}\right|\left(-\eta_{2}+\left(h_{2}+p_{2}\right)\right) \leq 0
$$

Thus the controller is also stable.

\section{Simulation Analysis}

Simulation parameters are listed in Table 1 . The initial value of the system is $(\alpha, \beta)=\left(0^{\circ}, 0^{\circ}\right)$, and the command is $\left(\alpha_{0}, \beta_{0}\right)=\left(1^{\circ}, 1^{\circ}\right)$. The parameters of controller are $k_{1}=0.5$ and $k_{2}=5$. Due to the diameter of a spinning missile, the ranges of the $\boldsymbol{u}$ are limited in $\left|\delta_{x}\right| \leq 0.2 m$ and $\left|\delta_{y}\right| \leq 0.2 m$. In the case of setting a fixed time of $0.5 \mathrm{~s}$, the simulation results are illustrated in Fig. 3 and Fig. 4. 
Table 1 Parameters of a spinning missile

\begin{tabular}{cccccccc}
\hline \hline$m_{s}[\mathrm{~kg}]$ & 100.00 & $J_{1}[\mathrm{~kg} \cdot \mathrm{m}]$ & 5.80 & $m_{B}[\mathrm{~kg}]$ & 88.00 & $\dot{\gamma}\left[\mathrm{rad} \cdot \mathrm{s}^{-1}\right]$ & 20.00 \\
\hline$m_{p}[\mathrm{~kg}]$ & 8.00 & $J_{2}[\mathrm{~kg} \cdot \mathrm{m}]$ & 51.40 & $S\left[\mathrm{~m}^{2}\right]$ & 0.05 & $\rho\left[\mathrm{kg} \cdot \mathrm{m}^{-3}\right]$ & 1.205 \\
\hline$m_{q}[\mathrm{~kg}]$ & 4.00 & $V\left[\mathrm{~m} \cdot \mathrm{s}^{-1}\right]$ & 5000.00 & $L[\mathrm{~m}]$ & 1.50 & $I[\mathrm{~m}]$ & 0.50 \\
\hline \hline
\end{tabular}

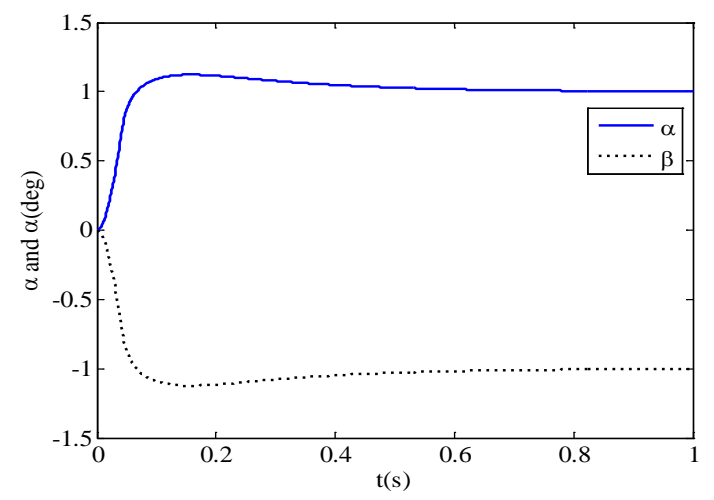

Fig. 3 The transition processes of the angle of attack and the sideslip angle

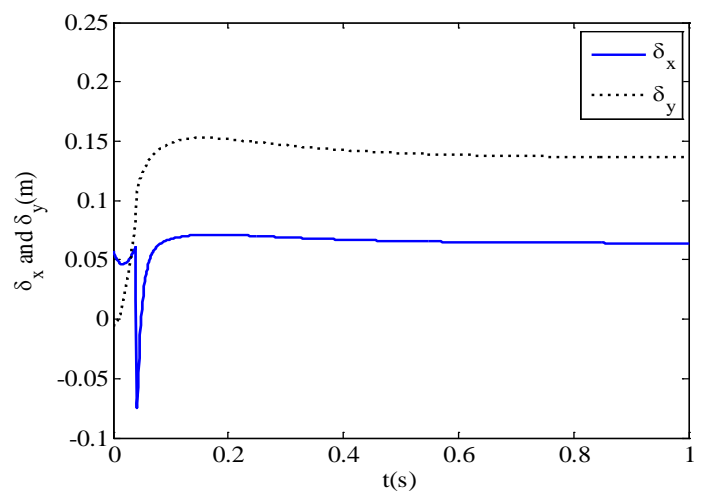

Fig. 4 The transition processes of the positions of moving masses

It is observed that control system of the hypersonic spinning missile can track the commands within 0.5 s which is given by designer. As shown in Fig.4, at the beginning of the control, $\delta_{x}$ has a peak caused by the disturbance. However, under the effect of the sliding mode controller, the peak can be quickly eliminated, and $\delta_{x}$ can also reach a steady state quickly.

\section{Conclusions}

The dynamics model of a hypersonic spinning missile with two moving masses is established. Following the backstepping approach, the control system is divided into two subsystems. An adaptive sliding mode control is used to design subsystem 1 , and a global fixed time convergent sliding mode surface is used to design subsystem 2 . The numerical simulations show that the attitude controller is effective, and the control system can track the commands within a given fixed time. However, the uncertainties of the flight parameters are difficult to describe accurately, and the further research should be focused on reducing the dependence of the controller on the uncertainties.

\section{Acknowledgements}

The grant support from the National Science Foundation of China (No. 11202023) is greatly acknowledged.

\section{References}

[1] Menon P K, Sweriduk G D. Integrated guidance and control of moving-mass actuated kinetic warheads. Journal of Guidance, Control and Dynamics, 2004, 27(1):118-126.

[2] Robinett R D, Sturgis B R, Kerr S A. Moving mass trim control for vehicles. Journal of Guidance, Control and Dynamics, 1996, 19(5): 1064-1070.

[3] LIAO Guo-bin, WAN Zi-ming. Study on the change of the roll tate and its effect on missile attitude for mass moment control missile. Aero Weapon, 2005, (5): 3-6.

[4] Woolsey C A. Reduced Hamiltonian dynamics for a rigid body coupled to a moving point mass. Journal of Guidance, Control and Dynamics, 2005, 28( 1): 131-138.

[5] GUO Peng-fei. Moving Mass Control and Terminal Guidance for Spinning Missiles. Beijing Institute of Technology, 2014. 